\title{
Stress analysis by local integral equations
}

\author{
V. Sladek ${ }^{1}$, J. Sladek ${ }^{1} \&$ Ch. Zhang $^{2}$ \\ ${ }^{1}$ Institute of Construction and Architecture, Slovak Academy of Sciences, \\ Bratislava, Slovakia \\ ${ }^{2}$ Department of Civil Engineering, University of Siegen, Germany
}

\begin{abstract}
This paper is a comparative study for various numerical implementations of local integral equations developed for stress analysis in plane elasticity of solids with functionally graded material coefficients. Besides two meshless implementations by the point interpolation method and the moving least squares approximation, the element based approximation is also utilized. The numerical stability, accuracy, convergence of accuracy and cost efficiency (assessed by CPU-times) are investigated in numerous test examples with exact benchmark solutions.

Keywords: elasticity, functionally graded materials, boundary value problems, force equilibrium, meshless implementations.
\end{abstract}

\section{Introduction}

A rapid progress can be observed in the development of various meshless techniques especially in fluid problems. Simultaneously, a considerable expansion of such techniques can be found also in various applications to engineering and science problems. This can be explained by the fact that there are known certain limitations of standard discretization techniques especially when applied to some classes of problems (e.g. problems in separable media, problems with free or moving boundaries; crack problems; problems with large distortions, etc.). Although the standard discretization techniques are applicable to the numerical solution of boundary value problems in continuously nonhomogeneous elastic media, the formulations developed for homogeneous media are not applicable directly, since the governing equations are now given by partial differential equations with variable coefficients.

There has not been a unique classification of meshless techniques up to now. Mostly they are classified according to the employed approximation. Some of 
the techniques utilize meshless approximation of field variables but a background mesh is still required for numerical integration especially in approaches based on global formulations. On the other hand, the local formulations bring a possibility to avoid the mesh completely with using nodes alone for approximation. Then, the physical principles can be formulated in integral forms on local sub-domains. A large group of meshless techniques are denoted as meshless local Petrov-Galerkin methods $[1,2]$ with bearing in mind that the Petrov-Galerkin weak form idea is applied in a local sense with selecting the trial and test functions independently and approximating the field variables in a meshless way.

Some comparative studies might be desired in this stage of rapid increase of literature devoted to various meshless techniques as well as their applications to various problems.

\section{Local integral equations}

Under assumption of static loading conditions, the demand of the force equilibrium in an arbitrary but small part of the elastic body results in the strong formulation of the governing equations given by the partial differential equations

$$
\sigma_{i j, j}(\mathbf{x})+X_{i}(\mathbf{x})=0 \text { in } \Omega,
$$

supplemented by the generalized Hooke's law

$$
\sigma_{i j}(\mathbf{x})=c_{i j k l}(\mathbf{x}) u_{k, l}(\mathbf{x}) .
$$

In the case of isotropic FGM, the spatial variation of the tensor of material coefficients is usually given via the variable Young's modulus as

$$
c_{i j k l}(\mathbf{x})=E(\mathbf{x}) c_{i j k l}^{o}, \quad c_{i j k l}^{o}=\frac{1}{2(1+v)}\left(\delta_{i k} \delta_{j l}+\delta_{i l} \delta_{j k}+\frac{2 \bar{v}}{1-2 \bar{v}} \delta_{i j} \delta_{k l}\right),
$$

with the material parameter $\bar{v}$ being expressed in terms of the constant Poisson ratio $v$ by

$$
\bar{v}=\left\{\begin{array}{lr}
v /(1+v), & \text { for plane stress } \text { conditions } \\
v, & \text { otherwise }
\end{array} .\right.
$$

Inserting (3) into (1), one obtains the governing PDE for displacements

$$
E(\mathbf{x}) c_{i j k l}^{o} u_{k, l j}(\mathbf{x})+E, j(\mathbf{x}) c_{i j k l}^{o} u_{k, l}(\mathbf{x})=-X_{i}(\mathbf{x}) .
$$

The standard boundary conditions prescribe a half of the boundary quantities $\left\{\tilde{u}_{i}(\boldsymbol{\eta}), \tilde{t}_{i}(\boldsymbol{\eta})\right\}$ for $(i=1, \ldots, d)$ at each boundary point $\boldsymbol{\eta} \in \partial \Omega$, with the traction vector being given by

$$
t_{i}(\boldsymbol{\eta})=n_{j}(\boldsymbol{\eta}) c_{i j k l}(\boldsymbol{\eta}) u_{k, l}(\boldsymbol{\eta}),
$$


where $n_{j}(\boldsymbol{\eta})$ denotes the Cartesian components of the unit outward normal vector on the boundary $\partial \Omega$.

In numerical formulations for solution of b.v.p., weak formulations are frequently utilized instead of the strong formulation. The governing equation is satisfied in a weak sense if the weighted integral of the governing equation is fulfilled

$$
\int_{\Omega}\left(\sigma_{i j, j}(\mathbf{x})+X_{i}(\mathbf{x})\right) w_{i k}(\mathbf{x}) d \Omega(\mathbf{x})=0 .
$$

Since the test (or weight) functions can be arbitrary, the weak formulation might have no physical interpretation. In order to apply the formulation with clear physical interpretation, we shall use the test functions given by the Heaviside functions with support on local sub-domains $\Omega^{c}$ of the whole analysed domain $\Omega$

$$
w_{i k}^{c}(\mathbf{x})=\left\{\begin{array}{l}
\delta_{i k}, \mathbf{x} \in \Omega^{c} \\
0, \quad \mathbf{x} \notin \Omega^{c}
\end{array} .\right.
$$

Then, the weak formulation (6) after using the Gauss divergence theorem yields the well known force equilibrium on local sub-domains $\Omega^{c}$

$$
\int_{\partial \Omega^{c}} n_{j}(\boldsymbol{\eta}) c_{i j k l}(\boldsymbol{\eta}) u_{k, l}(\boldsymbol{\eta}) d \Gamma(\boldsymbol{\eta})=-\int_{\Omega^{c}} X_{i}(\mathbf{x}) d \Omega(\mathbf{x}),
$$

which is the weak formulation with the clear physical interpretation.

Recall that the local integral equations (7) are non-singular, since there are no singular fundamental solutions involved in contrast to the singular integral equations employed in the boundary integral equation method. Moreover, the integration of unknown (approximated) field variables is constraint to the boundary of local sub-domains even in the case of non-homogeneous media. This can be effectively utilized by decreasing the amount of integration points as compared with the formulations involving the domain integrals.

\section{Numerical implementations}

In numerical solving, in general, the amount of degrees of freedom is decreased from infinity to a finite number by approximating the field variable in terms of certain shape functions and nodal unknowns. The nodal unknowns are determined by the set of equations obtained by collocating the prescribed boundary conditions at boundary nodes and force equilibrium equations at interior nodal points.

We shall consider domain-type approximations $u_{i}^{h}(\mathbf{x})$ for the displacements $u_{i}(\mathbf{x})$ within a sub-domain $\Omega_{s} \subset(\Omega \cup \partial \Omega)$. Then, it is possible to get also the approximations for displacement gradients by differentiating the approximated displacements. Thus, the discretized equations take the form 


$$
\begin{gathered}
u_{i}^{h}(\zeta)=\tilde{u}_{i}(\zeta) \text { at } \zeta \in \partial \Omega \text { where } \tilde{u}_{i}(\zeta) \text { is prescribed } \\
n_{j}(\zeta) c_{i j k l}(\zeta) u_{k, l}^{h}(\zeta)=\tilde{t}_{i}(\zeta) \text { at } \zeta \in \partial \Omega \text { where } \tilde{t}_{i}(\zeta) \text { is prescribed } \\
\int_{\partial \Omega^{c}} n_{j}(\boldsymbol{\eta}) c_{i j k l}(\boldsymbol{\eta}) u_{k, l}^{h}(\boldsymbol{\eta}) d \Gamma(\boldsymbol{\eta})=-\int_{\Omega^{c}} X_{i}(\mathbf{x}) d \Omega(\mathbf{x})
\end{gathered}
$$

on sub-domains $\Omega^{c}$ around interior nodes $\mathbf{y}^{c}$.

\subsection{Quadrilateral quadratic elements}

A 2-d plane domain $\Omega$ is assumed to be subdivided into $m$ conforming quadrilateral serendipity elements $S_{e}$ [3] with quadratic polynomial interpolation for the approximation of both the geometry and displacements. Then,

$$
\Omega=\bigcup_{e=1}^{m} S_{e},\left.\quad x_{i}\right|_{S_{e}}=\sum_{a=1}^{8} x_{i}^{a e} N^{a}\left(\xi_{1}, \xi_{2}\right),\left.\quad u_{i}^{h}(\mathbf{x})\right|_{S_{e}}=\sum_{a=1}^{8} u_{i}\left(\mathbf{x}^{a e}\right) N^{a}\left(\xi_{1}, \xi_{2}\right),
$$

where $x_{i}^{a e}$ are the Cartesian coordinates of the $a$-th nodal point on $S_{e}$ and $N^{a}$ represent the shape functions. Since the interpolation polynomials are expressed as functions of intrinsic coordinates, the expressions for displacement gradients are not trivial [4] and integrations are to be carried out in the transformed intrinsic space. The local sub-domain $\Omega^{c}$ is specified as union of elements adjacent to the interior node $\mathbf{y}^{c}$.

\subsection{Point interpolation method (PIM)}

As in all meshless approximation techniques, the shape functions derived for the approximation of the field variable $u_{i}(\mathbf{x})$ within a sub-domain $\Omega_{s}$ utilize only nodes scattered arbitrarily in the analyzed domain without any predefined mesh to provide a connectivity of the nodes.

Without going into details $[5,6]$, we present the interpolation formula for displacements in surroundings of the nodal point $\mathbf{x}^{q}$ in terms of the shape functions and nodal values as

$$
\left.u_{i}^{h}(\mathbf{x})\right|_{\Omega^{q}}=\sum_{\alpha=1}^{N^{q}} u_{i}\left(\mathbf{x}^{n(q, \alpha)}\right) \varphi^{(q, \alpha)}(\mathbf{x}),
$$

where $n(q, \alpha)$ stands for the global number of nodes from the interpolation domain $\Omega_{i}^{q}$. If $\Omega_{i}^{q}$ is defined as a circle with the radius $r^{q}$ the number of nodes involved in $\Omega_{i}^{q}$ is given as $N^{q}=\sum_{a=1}^{N_{t}} H\left(r^{q}-\left|\mathbf{x}^{a}-\mathbf{x}^{q}\right|\right)$, 
where $H(z)$ is the Heaviside unit step function and $N_{t}$ is the total number of nodes. The numerically stable development of the shape functions can be achieved by combining the polynomials and RBFs as basis functions in a $\mathrm{PIM}(\mathrm{P}+\mathrm{RBF})$ approach $[5,6]$. The explicit expression for the shape functions being given elsewhere [6]. Recall that the Kronecker-delta property is satisfied

$$
\varphi^{(q, \alpha)}\left(\mathbf{x}^{n(q, \beta)}\right)=\delta_{\alpha \beta} \text {. }
$$

Finally, the displacement gradients are approximated as gradients of approximated displacements

$$
\left.u_{i, j}^{h}(\mathbf{x})\right|_{\Omega^{q}}=\sum_{\alpha=1}^{N^{q}} u_{i}\left(\mathbf{x}^{n(q, \alpha)}\right) \varphi_{, j}^{(q, \alpha)}(\mathbf{x}),
$$

i.e., in terms of the nodal values of displacements and the derivatives of the shape functions.

Note that the shape functions and their derivatives are not available in closed form. Thus, certain computational algorithm is to be repeated at each evaluation point. Nevertheless, in the present formulation, some of the inverse matrices can be pre-computed and stored in the memory for each nodal point in order to save CPU-time.

\subsection{Moving least squares (MLS) approximation}

The MLS-approximation is widely used in meshless methods. The displacements are approximated in terms of certain shape functions and nodal unknowns as

$$
u_{i}^{h}(\mathbf{x})=\sum_{a=1}^{N_{t}} \phi^{a}(\mathbf{x}) \hat{u}_{i}^{a} .
$$

The shape functions are expressed in terms monomial basis functions and weights associated with each nodal point. They have to be computed according to certain algorithm at each evaluation point. Since the shape functions do not possess the Kronecker delta property, $\phi^{a}\left(\mathbf{x}^{b}\right) \neq \delta_{a b}$, in general, the nodal unknowns are expansion coefficients (fictitious nodal displacements) which are different from the actual nodal values of displacements.

Since the number of nodal points which contribute to the sum in Eq. (12) is controlled by the weights, one has to consider all the nodes in the summation. To decrease the amount of the considered nodes, the Central Approximation Node (CAN) concept can be used. Then, the number of considered nodes in each evaluation at $\mathbf{x}$ is reduced from $N_{t}$ to $N^{q}$, where $N^{q}<N_{t}$ is the number of nodes supporting the approximation at the central approximation node $\mathbf{x}^{q}$. The nodes supporting the approximation with the CAN located at $\mathbf{x}^{q}$ lie in the $\Omega_{C A N}^{q}$ specified by the radius $r^{q}$. Then, instead of the approximation given by Eq. (12), one can use 


$$
u_{i}^{h}(\mathbf{x})=\sum_{a=1}^{N^{q}} \hat{u}_{i}^{n(q, a)} \phi^{n(q, a)}(\mathbf{x}) .
$$

The gradients of displacements are approximated as gradients of approximated displacements given by Eqs. (12) and (13).

In contrast to the implementation based on finite elements, the integration in meshless approaches is carried out in the global Cartesian coordinate system. The choice of simple shape for sub-domains yields simple integration. The Gaussian quadrature proved to be more convenient than the trapezoidal rule for integration over the boundary of circular sub-domains, since the later exhibits very slow improvement of accuracy with fining the subdivision of the integration interval, what results in enormous increasing the computational time needed for evaluation of shape functions.

\section{Numerical tests}

In order to test the proposed numerical methods, we consider examples for which analytical solutions are available. The body forces are vanishing in $\Omega$, the Poisson ratio is constant $v=0.25$, plane stress conditions are assumed and for conciseness, we present only the numerical results for exponential gradation $E(\mathbf{x})=E_{o} \exp \left(2 \delta x_{2} / L\right)$ with $\delta=3$. The considered domain is a square $L \times L$

with applied tension load on the top, fixed bottom in vertical direction and tractions on the lateral sides are given by the analytical solution [7].

In the study of the convergence and accuracy of the numerical results with respect to the increasing density of nodal points, we use the displacement norm $\%$ error defined as

$$
\begin{gathered}
\text { displ. norm error }(\%)=100\left\{\begin{array}{c}
N_{t} \\
\sum_{a=1} \Delta u_{i}^{a} \Delta u_{i}^{a}
\end{array}\right\}^{1 / 2} /\left\{\sum_{a=1}^{N_{t}} u_{i}^{e x}\left(\mathbf{x}^{a}\right) u_{i}^{e x}\left(\mathbf{x}^{a}\right)\right\}^{1 / 2} \\
\Delta u_{i}^{a}=u_{i}^{n u m}\left(\mathbf{x}^{a}\right)-u_{i}^{e x}\left(\mathbf{x}^{a}\right),
\end{gathered}
$$

where $N_{t}$ is the total number of nodes on the closed domain $\Omega \cup \partial \Omega$.

In most of the presented computations, we shall use a homogeneous distribution of nodes with $h^{a}=\min _{\forall b}\left\{\left|\mathbf{x}^{a}-\mathbf{x}^{b}\right|\right\}=$ const $=h$.

In the PIM, we have used combination of polynomial functions (given by six monomial basis) with RBFs for which we considered multiquadrics, Gaussian RBFs, and the 8-order spline. Similar in the case of MLS-approximation, we have used three different kinds of weights given by Gaussian, exponential, and 8-order spline weights. Although the shape and size of sub-domains can be chosen arbitrarily, the results of numerical computations may depend on these aspects and similarly on the shape parameters involved in both the RBFs and 
weights of the MLS-approximation. Therefore, firstly we have investigated the stability of numerical computations with respect to those three indicators. The use of square shape for sub-domains yields better results as compared with the circular shape. In the next computations, we used optimal values for the shape parameter and the size of sub-domains which guarantee the numerical stability. The numerical instability with respect to acceptable accuracy was observed in case of exponential weights used in MLS-approximation. The CAN concept with the nearest node to the evaluation point proved to give the best results in both the meshless techniques. The radius of the interpolation domain is taken as

$$
r^{a}=3.001 h^{a} \text {. }
$$

Fig. 1 shows the convergence of the numerical solutions by various $\mathrm{PIM}(\mathrm{P}+\mathrm{RBF})$ approaches. The increasing density of nodes is represented by the decreasing parameter $h / L$. Fig.2 illustrates the variation of the displacement field $u_{2}\left(L / 2, x_{2}\right)$ along the vertical line $\left(L / 2, x_{2}\right)$ with $x_{2} \in[0, L / 2]$. It can be seen that excellent accuracy is achieved even in the case of strong gradation of Young's modulus when the variation of displacements differs dramatically from the case of homogeneous medium. The accuracy of numerically computed interior stresses is also reasonable (the results will be summarized in Tab.1). Thus, the PIM based on the combination of polynomials and the multiquadrics with $m=5 / 2$ seems to be appropriate even for strong non-homogeneity $\delta=3$, when the Young modulus on the top of the square domain is 403 times higher than on the bottom.

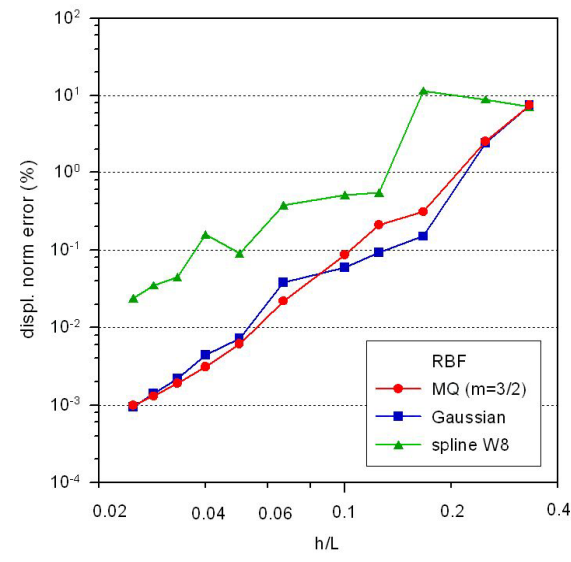

Figure 1: Convergence study.

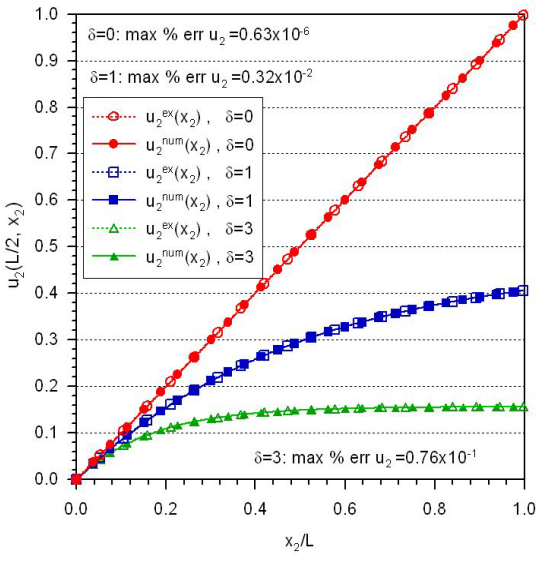

Figure 2: Displacement results.

It is interesting to compare the results by two variants of the MLSapproximation: standard formulation vs CAN-nearest node concept. Fig. 3 shows the comparison of the convergence of numerical results by using these two different approaches with Gaussian weights. It can be seen that the accuracy is almost invariant with respect to the predefinition of supporting nodes. On the other hand, the influence on the CPU-times is much more significant (Fig. 4). Although we can see negligible differences between the CPU-times resulting 
from both the formulations provided that the densities of nodes are low, the rates for the CPU-time increase are significantly different for each of the employed approaches. The difference between the CPU-times by the CAN-nearest node approach and standard MLS approach is increasing remarkably with increasing the density of nodes.

Finally, we present some comparisons of the best formulations based on the use of three different kinds of domain-type approximations. The best meshless formulations utilize the CAN-nearest node concept and are characterized by selection of square shapes for sub-domains, and optimal values of the shape parameter (involved in RBFs and/or weights) as well as the sub-domain size parameter.

The QE-approach exhibits reliable convergence of accuracy with increasing the density of nodes, but lower accuracy is achieved in the FGM sample with strong gradation of the Young modulus $(\delta=3)$ as compared with the meshless PIM and/or MLS results (Fig. 5).
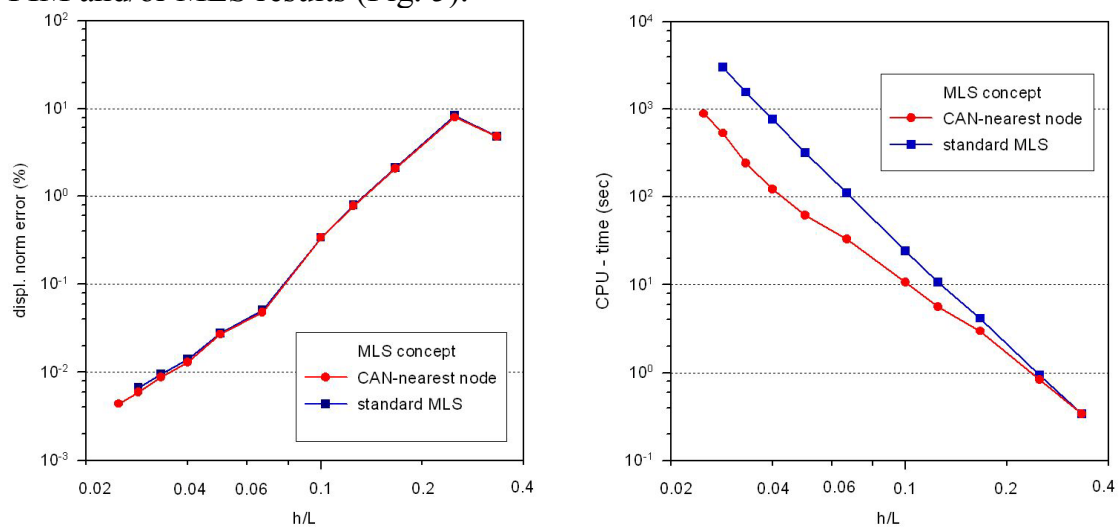

Figure 3: Accuracies by two MLS Figure 4: CPU by two MLS concepts. concepts.

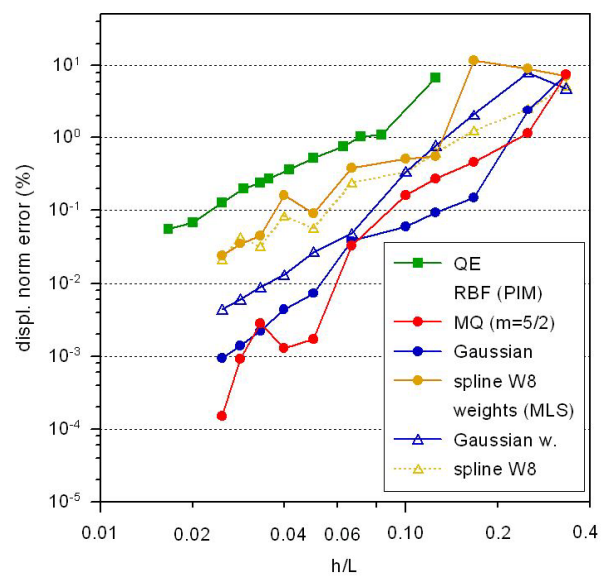

Figure 5: Comparison of accuracies by various numerical techniques. 
A comparison of the CPU-times by the QE-approach with various meshless approaches is given in Fig. 6. The CPU-times by meshless approaches converge to each other by increasing the density of nodes and the differences between the $\mathrm{QE}$ and meshless approaches are diminished. This can be explained by the fact that with increasing the amount of nodes the time needed for solution of the system of discretized equations is becoming dominant in comparison with the time needed for evaluation of the system matrix.

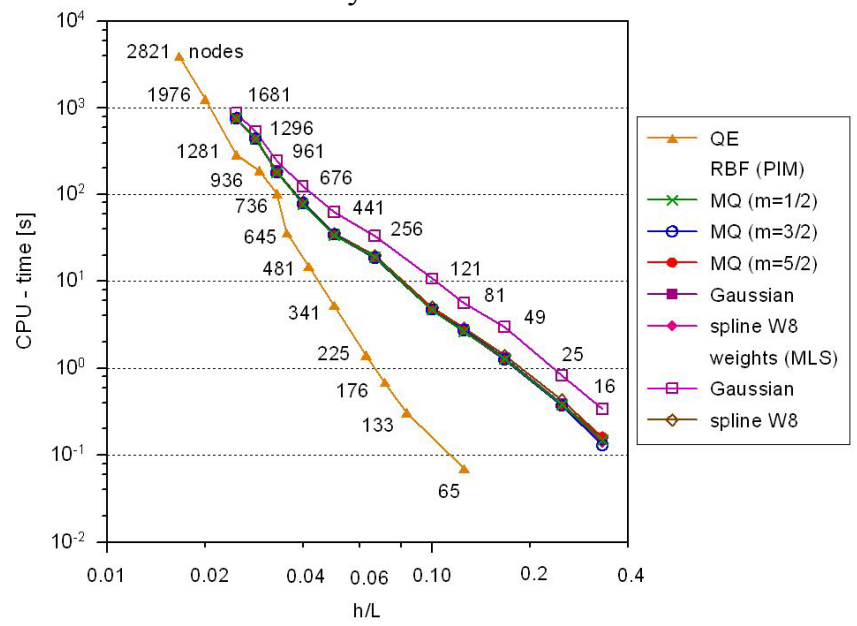

Figure 6: Comparison of CPU-times by various numerical techniques.

Table 1: $\quad$ Maximal \% errors for displacements and stresses computed at interior points along the vertical line $\left(L / 2, x_{2}\right)$ in square sample.

\begin{tabular}{|c|c|c|c|c|c|}
\hline \multirow{2}{*}{$\begin{array}{l}\text { computational } \\
\text { method }\end{array}$} & \multirow{2}{*}{$\begin{array}{c}\text { grade } \\
\text { parameter }\end{array}$} & \multicolumn{3}{|c|}{$\max \%$ error } & \multirow{2}{*}{$\begin{array}{l}\mathrm{CPU} \\
{[\mathrm{sec}]}\end{array}$} \\
\hline & & $u_{2}$ & $\sigma_{22}$ & $\sigma_{11}$ & \\
\hline \multirow{3}{*}{$\begin{array}{l}\text { LIE-QE } \\
\text { (400 elem.) } \\
1281 \text { nodes }\end{array}$} & $\delta=0$ & $0.84 \times 10^{-10}$ & $0.39 \times 10^{-9}$ & - & \multirow{3}{*}{290} \\
\hline & $\delta=1$ & 0.099 & 0.18 & 0.31 & \\
\hline & $\delta=3$ & 0.93 & 1.93 & 5.83 & \\
\hline \multirow{3}{*}{$\begin{array}{c}\text { LIE- } \\
\text { PIM(P+MQ) } \\
\text { (441 nodes) }\end{array}$} & $\delta=0$ & $0.63 \times 10^{-6}$ & $0.88 \times 10^{-6}$ & - & \multirow{3}{*}{35} \\
\hline & $\delta=1$ & 0.0032 & 0.0083 & 0.032 & \\
\hline & $\delta=3$ & 0.076 & 0.22 & 3.77 & \\
\hline \multirow{3}{*}{$\begin{array}{l}\text { LIE-MLS } \\
\text { (441 nodes) }\end{array}$} & $\delta=0$ & $0.10 \times 10^{-9}$ & $0.57 \times 10^{-9}$ & - & \multirow{3}{*}{65} \\
\hline & $\delta=1$ & 0.11 & 0.16 & 0.20 & \\
\hline & $\delta=3$ & 0.78 & 1.54 & 1.68 & \\
\hline
\end{tabular}


The slightly higher relative error for the stresses $\sigma_{11}$ is due to small value of this component in the considered b.v.p.

Similar results have been obtained also for stress analysis in both the transversal and axial cross-section of the thick-wall tube.

\section{Conclusions}

Both the meshless techniques proved to be useful for numerical implementation of the LIEs applied to stress analysis problems even in the case of strong gradation of the Young modulus.

- Acceptable accuracy, convergence of accuracy and numerical stability are guaranteed by using the proposed techniques.

- Great savings in the CPU-time are achieved by using the CAN-nearest node concept.

- The accuracy by the QE-approach is slightly worse than by meshless approaches, but reliable convergence is achieved with increasing the density of nodes.

\section{Acknowledgements}

The research has been supported by the Slovak Grant Agencies VEGA, APVV and German Research Foundation (DFG), which are gratefully acknowledged.

\section{References}

[1] Atluri S.N., Shen S., The meshless local Petrov-Galerkin (MLPG) method, Tech Science Press: Encino, 2002.

[2] Atluri S.N., The meshless method (MLPG) for domain \& BIE discretizations, Tech Science Press: Forsyth, 2004.

[3] Hughes T.J.R., The Finite Element Method. Linear Static and Dynamic Finite Element Analysis. Prentice-Hall, Inc.: Englewood Cliffs, 1987.

[4] Sladek V., Sladek J., Zhang Ch., Local integro-differential equations with domain elements for numerical solution of PDE with variable coefficients. $J$. Eng. Mathematics 51, pp. 261-282, 2005.

[5] Liu G.R., Mesh Free Methods, Moving beyond the Finite Element Method. CRC Press: Boca Raton, 2003.

[6] Sladek V., Sladek J., Tanaka M., Local integral equations and two meshless polynomial interpolations with application to potential problems in nonhomogeneous media. Computer Modeling in Engineering \& Sciences 7, pp. 69-83, 2005.

[7] Sladek V., Sladek J., Zhang Ch., A meshless Point Interpolation Method for Local Integral Equations in elasticity of non-homogeneous media. Advances in the Meshless Method, eds. J. Sladek, V. Sladek, Tech Science Press: Forsyth, 2006. 